Internat. J. Math. \& Math. Sci.

Vol. 22, No. 1 (1999) 81-84

S $0161-1712\langle 99\rangle 22081-9$

(c) Electronic Publishing House

\title{
SOME REMARKS ON THE ALGEBRAIC STRUCTURE OF THE FINITE COXETER GROUP $F_{4}$
}

\author{
MUHAMMAD A. ALBAR and NORAH AL-SALEH
}

(Received 10 October 1996 and in revised form 31 January 1997)

ABSTRACT. We consider in this paper the algebraic structure and some properties of the finite Coxeter group $F_{4}$.

Keywords and phrases. Presentation, Reidemeister-Schreier method, Coxeter groups.

1991 Mathematics Subject Classification. 20F05.

1. Introduction. The group $F_{4}$ is one of the irreducible Coxeter groups [9] defined by the presentation

$$
\begin{aligned}
& F_{4}=\left\langle x_{1}, x_{2}, x_{3}, x_{4}\right| x_{i}^{2}=e, \quad 1 \leq i \leq 4 \\
&\left.\left(x_{1} x_{2}\right)^{3}=\left(x_{3} x_{4}\right)^{3}=\left(x_{2} x_{3}\right)^{4}=\left(x_{1} x_{3}\right)^{2}=\left(x_{1} x_{4}\right)^{2}=\left(x_{2} x_{4}\right)^{2}=e\right\rangle .
\end{aligned}
$$

It has the graph

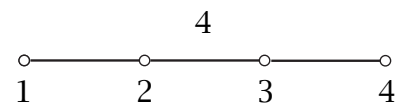

It is obvious that the group $B_{3}$ whose graph is

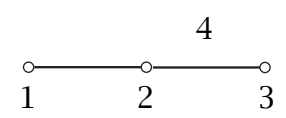

is a subgroup of $F_{4}$. The order of $B_{3}$ is known to be 48 [4]. It is easy to see that the index of $B_{3}$ in $F_{4}$ is 24 and hence the order of $F_{4}$ is 1152 .

2. The structure of $F_{4}$. We define $F_{4}$ by the presentation given in Section 1 . We consider the symmetric group of degree 3 with the presentation

$$
S_{3}=\left\langle x, y \mid x^{2}=y^{2}=(x y)^{3}=e\right\rangle .
$$

We define the map $\theta: F_{4} \longrightarrow S_{3}$, where

$$
\theta\left(x_{1}\right)=x, \quad \theta\left(x_{2}\right)=y, \quad \theta\left(x_{3}\right)=\theta\left(x_{4}\right)=e .
$$

It is easy to see that $\theta$ is an epimorphism and so $F_{4} / \operatorname{ker} \theta \cong S_{3}$. We use the Reidemeister-Schreier process to find a partition for $\operatorname{ker} \theta$. 
A Schreier transversal for $\operatorname{ker} \theta$ in $F_{4}$ is

$$
U=\left\{e, x_{1}, x_{2}, x_{1} x_{2}, x_{2} x_{1}, x_{1} x_{2} x_{1}\right\} .
$$

The process gives us the following partition for $\operatorname{ker} \theta$ :

$$
\begin{aligned}
\operatorname{ker} \theta=\langle a, b, c, d| a^{2}=b^{2}=c^{2}=d^{2}= & (a b)^{2}=(b c)^{2} \\
& \left.=(a d)^{3}=(b d)^{3}=(c d)^{3}=(a c)^{2}=e\right\rangle .
\end{aligned}
$$

Therefore, $\operatorname{ker} \theta$ is the Coxeter group $D_{4}$ whose graph is

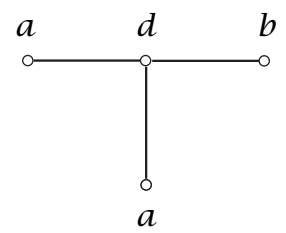

This shows that the group $F_{4}$ is the split extension of the Coxeter group $D_{4}$ by $S_{3}$.

REMARK 1. To identify the structure of $D_{4}$, we consider the map $\theta: D_{4} \rightarrow S_{4}$, where $D_{4}$ is defined by the graph above and $S_{4}$ is defined by the graph

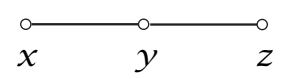

and $\theta(a)=x, \theta(d)=y, \theta(b)=z$, and $\theta(c)=y$. Using the Reidemeister-Schreier process, we find that ker $\theta \cong Z_{2}^{3}$. Thus, $D_{4}$ is the split extension of $Z_{2}^{3}$ by $S_{4}$. An alternative method is given in [3], where $D_{n}$ is shown to be the semi-direct product of $Z_{2}^{n-1}$ by $S_{n}$.

REMARK 2. A third method to show that $F \cong D_{4} \rtimes S_{3}$ follows. We consider $D_{4}$ and $S_{3}$ as having the following graphs:
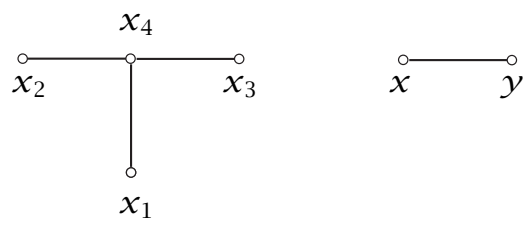

where $x=(12)$ and $y=(23)$. We consider the natural action of $S_{3}$ or $D_{4}$ defined as

$$
\left(x_{1}, x_{2}, x_{3}, x_{4}\right)^{x}=\left(x_{2}, x_{1}, x_{3}, x_{4}\right) \text { and }\left(x_{1}, x_{2}, x_{3}, x_{4}\right)^{y}=\left(x_{1}, x_{3}, x_{2}, x_{4}\right) .
$$

We let $E$ to be the split extension of $D_{4}$ by $S_{3}$ with this action. A presentation for $E$ is

$$
\left.E=\left\langle x_{1}, x_{2}, x_{3}, x_{4}, x, y\right| \text { Relations of } D_{4} \text {, Relations of } S_{3} \text {, Action of } S_{3} \text { on } D_{4}\right\rangle .
$$

(See [2].) Simple Tietze transformations show that $E \cong F_{4}$. Hence, $F_{4} \cong D_{4} \rtimes S_{3}$. 
3. The derived series of $F_{4}$. We use the Reidemeister-Schreier process several times to find the derived series of $F_{4}$. Firstly, let $F_{4}$ have the presentation in Section 1. $F_{4} / F_{4}^{\prime} \cong Z_{2} \times Z_{2}$ and we find that $F_{4}^{\prime}=\left\langle x, y \mid x^{3}=y^{3}=\left(x^{-1} y^{-1} x y\right)^{2}=e\right\rangle$. The group $F_{4}^{\prime} / F_{4}^{\prime \prime} \cong Z_{3} \times Z_{3}$ and we get $F_{4}^{\prime \prime}=\langle a, b, c, d| a^{2}=b^{2}=c^{2}=d^{2}=(a b)^{2}=(a c)^{2}=$ $\left.(c d)^{2}=(b d)^{2}=(b d c a)^{2}=e\right\rangle$. Finally, $F_{4}^{\prime \prime} / F_{4}^{\prime \prime \prime} \cong Z_{2}^{4}$ and we find $F_{4}^{\prime \prime \prime}=Z_{2}$. Thus, we have proved that $F_{4}$ is solvable of derived length 4 .

4. The center and the growth series of $F_{4}$. We have seen in Section 2 that $F_{4} \cong$ $D_{4} \rtimes S_{3}$ and that $D_{4} \cong Z_{2}^{3} \rtimes S_{4}$. It is easy to see that the center of $D_{4}$ is $Z_{2}$ (in general, $Z\left(D_{n}\right)=Z_{2}$ if $n$ is even and $\{e\}$ if $n$ is odd [3]). Since $Z\left(S_{3}\right)=\{e\}$, we see that $Z\left(F_{4}\right) \subseteq$ $Z\left(D_{4}\right)=Z_{2}$. Let $Z\left(D_{4}\right)$ be generated by $g$. From the Reidemeister-Schreier process, we can find $g$ in terms of the generators of $F_{4}$ and show that it does not commute with any of them. Hence, $Z\left(F_{4}\right)=\{e\}$.

The growth series (in the sense of Gromov and Milnor) of $F_{4}$ is [5]

$$
\gamma\left(F_{4}\right)=(1+t)^{4}\left(1+t^{2}\right)^{2}\left(1+t^{4}\right)\left(1-t+t^{2}\right)^{2}\left(1+t+t^{2}\right)^{2}\left(1-t^{2}+t^{4}\right) .
$$

The order of $F_{4}$ is obtained here as $\gamma\left(F_{4}\right)(1)=2^{4} \times 2^{2} \times 2 \times 3^{2}=1152$.

ACKNOwLedgement. The first author thanks King Fahd University of Petroleum and Minerals for the support he has got to conduct this research.

\section{REFERENCES}

[1] N. A. Al Saleh, On the finite Coxeter groups, Ph.D. thesis, College of Girls, Dammam, Saudia Arabia, 1994.

[2] M. A. Albar, On presentation of group extensions, Comm. Algebra 12 (1984), no. 23-24, 2967-2975. MR 86g:20040. Zbl 551.20017.

[3] M. A. Albar and N. A. Al Saleh, The Coxeter group $D_{n}$, submitted.

[4] _ On the affine Weyl group of type $B_{n}$, Math. Japon. 35 (1990), no. 4, 599-602. MR 91d:20030. Zbl 790.20048.

[5] M. A. Albar, N. A. Al Saleh, and M. A. Al Hamed, The growth series of Coxeter groups, 47 (1998), no. 3, 417-428.

[6] C. T. Benson and L. C. Grove, Finite reflection groups, Bogden \& Quigley, Inc., Publishers, Tarrytown on Hudson, N.Y., 1971. MR 52 4099. Zbl 579.20045.

[7] N. Bourbaki, Elements de mathematique. Groupes et algebres de Lie, Actualites Scientifiques et Industrielles, no. 1337, Hermann, Paris, 1968 (French), Chapitre IV: Groupes de Coxeter et systemes de Tits. Chapitre V: Groupes engendres par des reflexions. Chapitre VI: systemes de racines. MR 39\#1590. Zbl 186.33001.

[8] N. Broderick and G. Maxwell, The crystallography of Coxeter groups. II, J. Algebra 44 (1977), no. 1, 290-318. MR 58 11162b. Zbl 348.20041.

[9] H. S. M. Coxeter and W. O. J. Moser, Generators and relations for discrete groups, fourth ed., Ergebnisse der Mathematik und ihrer Grenzgebiete [Results in Mathematics and Related Areas], vol. 14, Springer-Verlag, Berlin,New York, 1980. MR 81a:20001. Zbl 422.20001.

[10] J. E. Humphreys, Reflection groups and Coxeter groups, Cambridge Studies in Advanced Mathematics, vol. 29, Cambridge University Press, Cambridge, 1990. MR 92h:20002. Zbl 768.20016.

[11] G. Maxwell, The crystallography of Coxeter groups, J. Algebra 35 (1975), 159-177. MR 58 11162a. Zbl 312.20029.

[12] M. Suzuki, Group theory. I, Grun1dlehren der Mathematischen Wissenschaften [Fundamental Principles of Mathematical Sciences], vol. 247, Springer-Verlag, Berlin, 
New York, 1982, Translated from the Japanese by the author. MR 82k:20001c. Zbl 472.20001 .

Albar: Department of Mathematical Sciences, King Fahd University of Petroleum AND MiNERALS, DHAHRAN, 31261, SAUDI ARABIA

Al-SAleh: Department of Mathematics, College of Girls, DAmmam, SAUdi Arabia 


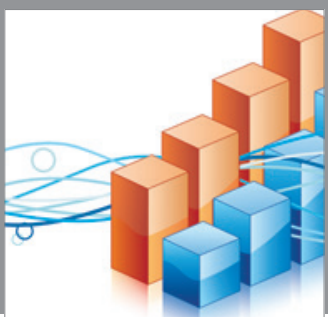

Advances in

Operations Research

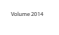

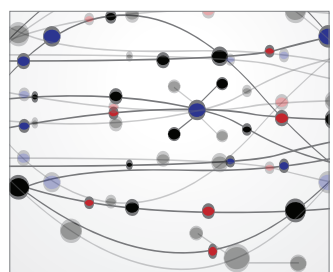

\section{The Scientific} World Journal
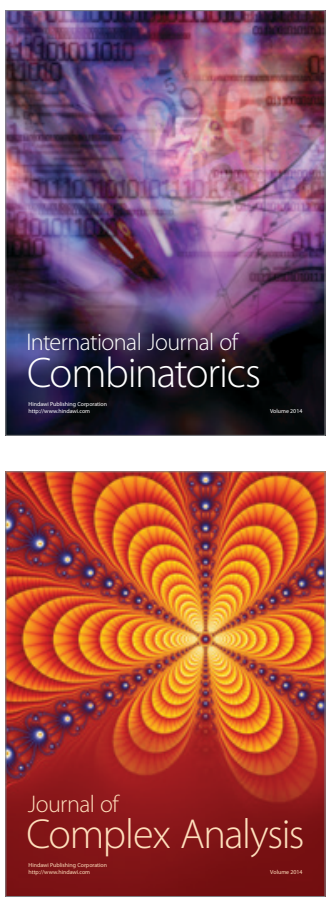

International Journal of

Mathematics and

Mathematical

Sciences
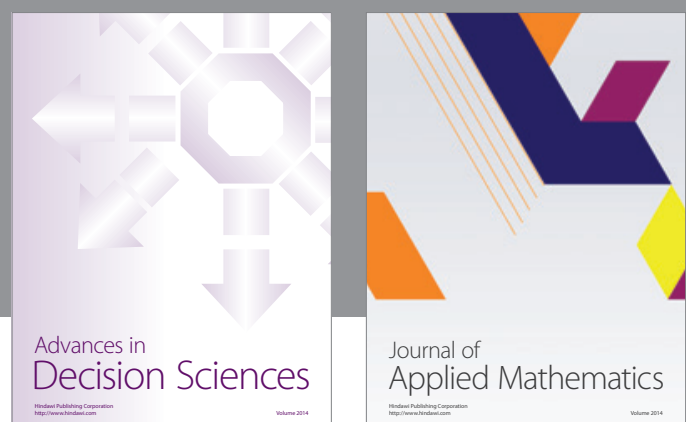

Journal of

Applied Mathematics
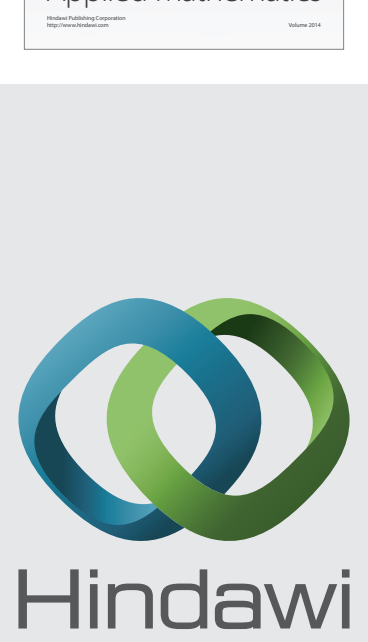

Submit your manuscripts at http://www.hindawi.com
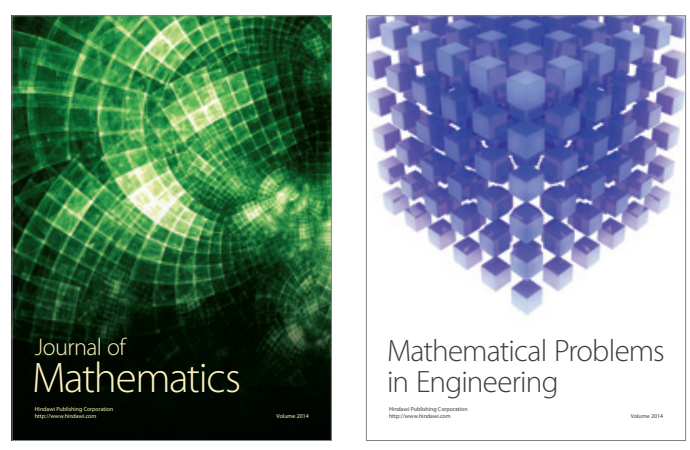

Mathematical Problems in Engineering
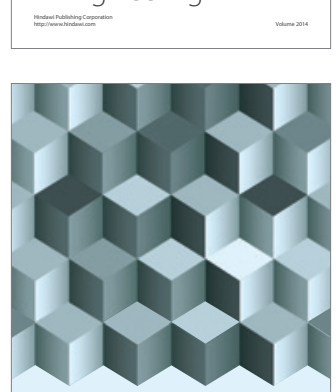

Journal of

Function Spaces
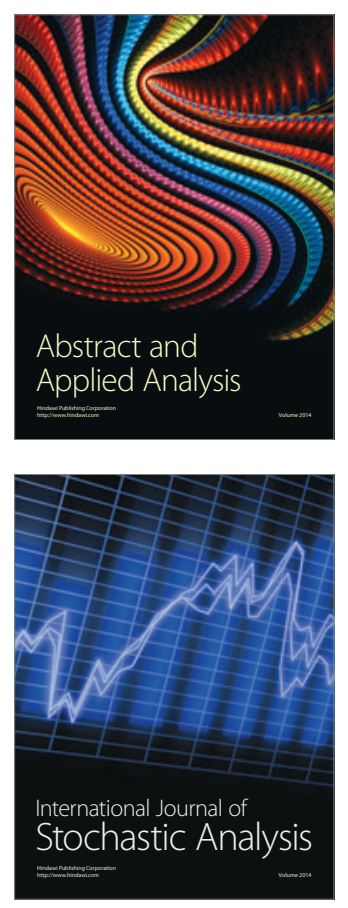

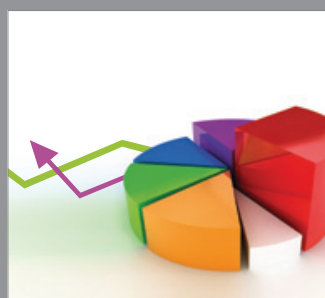

ournal of

Probability and Statistics

Promensencen
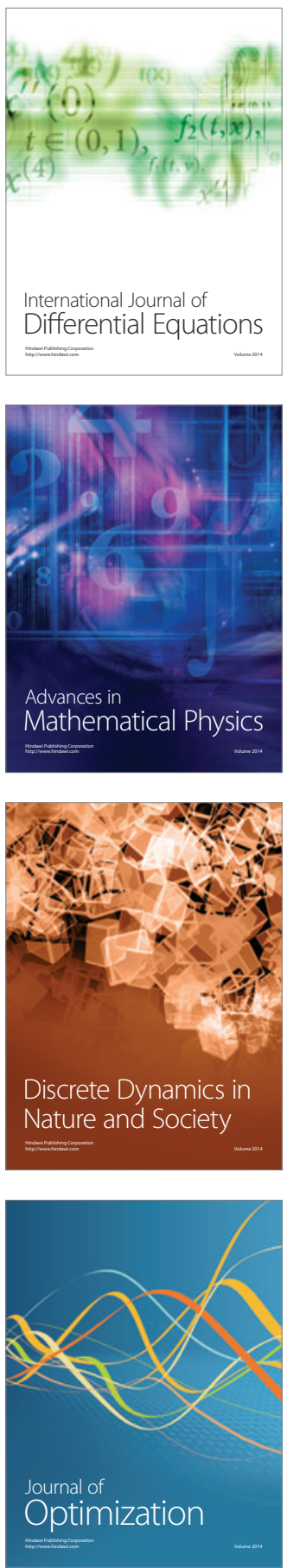